\title{
AIP
}

\section{Rotational dynamics of a dipolar supercooled liquid}

Gemma Sesé, Jordi Ortiz de Urbina, and Ricardo Palomar

Citation: J. Chem. Phys. 137, 114502 (2012); doi: 10.1063/1.4752426

View online: http://dx.doi.org/10.1063/1.4752426

View Table of Contents: http://jcp.aip.org/resource/1/JCPSA6/v137/i11

Published by the AIP Publishing LLC.

\section{Additional information on J. Chem. Phys.}

Journal Homepage: http://jcp.aip.org/

Journal Information: http://jcp.aip.org/about/about_the_journal

Top downloads: http://jcp.aip.org/features/most_downloaded

Information for Authors: http://jcp.aip.org/authors

\section{ADVERTISEMENT}

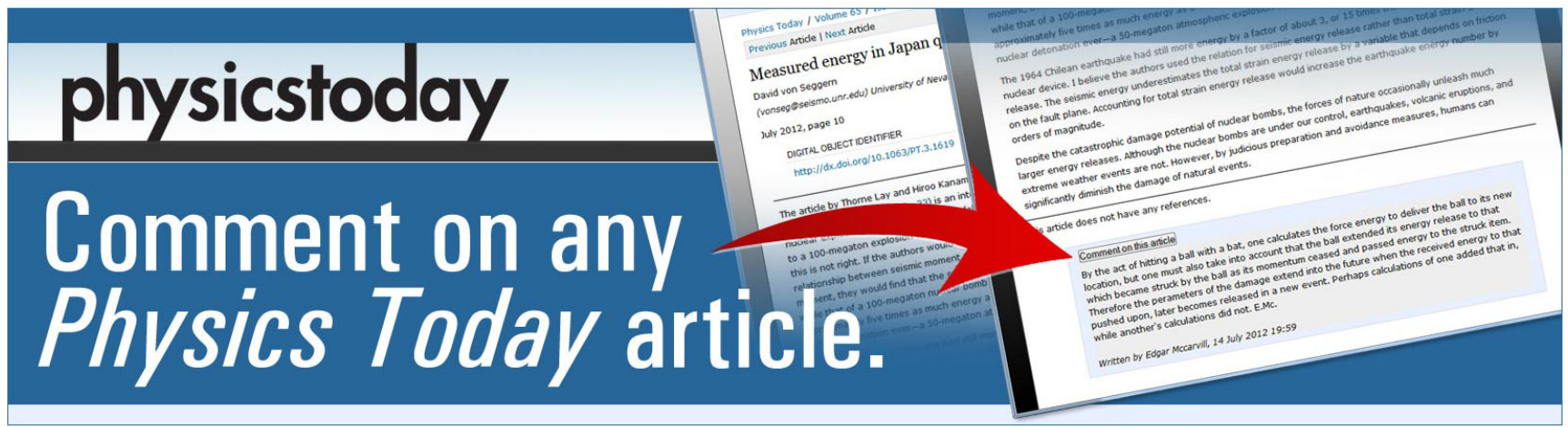




\title{
Rotational dynamics of a dipolar supercooled liquid
}

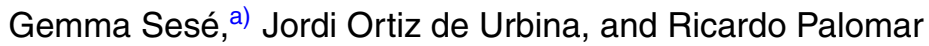 \\ Departament de Física i Enginyeria Nuclear, Universitat Politècnica de Catalunya, Campus Nord-Mòdul B4, \\ c/ Jordi Girona 1-3, 08034 Barcelona, Spain
}

(Received 28 June 2012; accepted 30 August 2012; published online 17 September 2012)

\begin{abstract}
We study the rotational dynamics of a supercooled molecular liquid by means of molecular dynamics simulations. The system under investigation is composed of rigid diatomic molecules with an associate dipole moment. At room temperature, orientational correlations decrease rapidly with increasing distances. Upon cooling, angles between dipole moments of molecules within the first coordination shell decrease. As for the dynamical properties, rotational diffusion coefficients decrease with temperature at a smaller rate than translational diffusion coefficients do, and the critical temperature associated with the former is lower than the one corresponding to their translational counterparts. Translation and rotation about an inertial axis are uncorrelated, whereas some coupling between translation and dipole reorientation is obtained. (C) 2012 American Institute of Physics. [http://dx.doi.org/10.1063/1.4752426]
\end{abstract}

\section{INTRODUCTION}

When molecular liquids are rapidly cooled, their dynamics experiences a tremendous slowing down. Experimental work has revealed that translational and rotational dynamics are not equally affected when approaching the glass transition. ${ }^{1}$ It has been obtained for some fragile liquids that rotational diffusion remains inversely proportional to viscosity at temperatures at which translational diffusion already displays a different scaling behaviour and a lower structural coupling. ${ }^{2,3}$ Moreover, in colloidal glasses, which are considered as experimental models to study the glass transition, a rotational glass transition has been recently observed, in addition to the translational one. ${ }^{4}$

Simulations play an important role in the fundamental understanding of supercooled liquids properties. Rotational dynamics and its connection with translation has been analyzed in simulations of several molecular glass formers as ortho-terphenyl ${ }^{5}$ and water, ${ }^{6,7}$ where a decoupling was encountered. ${ }^{8}$ For a model system of rigid dumbbells it has been found that jumps are important at temperatures close to the critical one, ${ }^{9}$ and that angular jumps are more relevant than translational ones in the deeply supercooled liquid. ${ }^{10}$ The analysis of the translational-rotational coupling in that nonpolar system has raised some questions on the adequacy of the diffusive model to describe rotational dynamics in the supercooled state. ${ }^{11}$

Our work is focused on the study of reorientational and rotational dynamics in a supercooled dipolar molecular liquid by means of molecular dynamics simulations. ${ }^{12}$ The molecular model has been built from the one of methanol molecules, but lacking sites for hydrogen bonding. Then, rigid diatomic molecules with an associate dipole moment are considered. Time correlation functions that characterize translational dynamics of the system have previously revealed the existence of three dynamic regimes at low temperatures: the ballistic

\footnotetext{
${ }^{a)}$ E-mail: gemma.sese@upc.es.
}

regime for short time intervals, the long-time regime or $\alpha$ relaxation, and the intermediate regime or $\beta$-relaxation, which becomes a plateau at very low temperatures. ${ }^{13}$ In the present work, we look at the molecular dipole reorientation and the rotation about a molecular inertial axis. Rotational diffusion coefficients are evaluated from the latter, and relaxation times for both processes are analyzed. We investigate their coupling with translation as well as the influence of structural orientational properties.

\section{MODELS AND SIMULATION DETAILS}

Molecular dynamics simulations are performed on a model system composed of rigid diatomic neutral molecules with an associate dipole moment. Molecules are slightly asymmetric, being 15 and 16 uma the masses of the molecular sites, which mimic the methyl group and the oxygen atom, respectively. Their charges are $0.323 e$ and $-0.323 e$. Then, the molecular dipole equals that of methanol molecules $(2.22 \mathrm{D})$, and the molecular mass is only $3 \%$ lower than that of methanol, but this molecular model is unable to establish hydrogen bonds. Molecular sites belonging to different molecules interact by means of electrostatic and LennardJones forces. Details on the intermolecular potential can be found elsewhere. ${ }^{14}$

The simulated system is made up of $N=1000$ molecules located in a cubic box with periodic boundary conditions. After being equilibrated at room temperature, the system has been quenched at constant pressure according to the procedure described in Ref. 14. For selected temperatures between $298 \mathrm{~K}$ and $103 \mathrm{~K}$, the samples have been equilibrated. Production runs of $1 \mathrm{~ns}$ at the highest temperature and of $6 \mathrm{~ns}$ at the lowest temperature have been performed in the $(N, V, T)$ ensemble.

Study of translation in this system has been previously focused on the dynamics of molecular centers-of-mass (COM). Analyses of time correlation functions such as mean 
TABLE I. Parameters obtained for the fits of relaxation times $\tau, \tau_{2} \propto(T$ $\left.-T_{c}\right)^{-\gamma}$ and of diffusion coefficients $D, D_{r} \propto\left(T-T_{c}\right)^{\gamma}$.

\begin{tabular}{lcccc}
\hline \hline & $\tau$ & $\tau_{2}$ & $D^{\text {a }}$ & $D_{r}$ \\
\hline$T_{c}$ & 96 & 98 & 94 & 58 \\
$\gamma$ & 1.9 & 1.2 & 2.2 & 1.8 \\
\hline \hline
\end{tabular}

a Reference 14.

square displacement and self-incoherent scattering function ${ }^{15}$ have been performed. Both functions display a three-step relaxation for the supercooled system, in good agreement with mode-coupling predictions. ${ }^{16}$ For their long-time regimes, it was possible to evaluate the diffusion coefficient $(D)^{17}$ and the translational relaxation time $(\tau),{ }^{18}$ respectively. After fitting both of them to a power law $\left(D, 1 / \tau \propto\left(T-T_{c}\right)^{\gamma}\right)$, the same value for the critical temperature was obtained $\left(T_{c}\right.$ $=95 \mathrm{~K}$ ), within statistical uncertainty. ${ }^{13,14}$ The corresponding fitting parameters have been gathered in Table I.

\section{RESULTS}

\section{A. Static orientational properties}

We investigate the orientation between different molecules by measuring the angle $\theta_{12}$ between $\mathbf{u}_{\mathbf{1}}$ and $\mathbf{u}_{2}$, which are unit vectors in the directions of the dipole moments of molecules 1 and 2, respectively,

$$
\theta_{12}(r)=\cos ^{-1}\left(\mathbf{u}_{2} \cdot \mathbf{u}_{1}\right) \text {. }
$$

$\theta_{12}$ strongly depends on intermolecular distance, as shown in Figure 1, where the function is plotted against $r=|\mathbf{r}|=\mid$ $\mathbf{r}_{\mathbf{c m} 2}-\mathbf{r}_{\mathbf{c m} 1} \mid$, being $\mathbf{r}_{\mathbf{c m} 1}$ and $\mathbf{r}_{\mathbf{c m} 2}$ the COM positions of molecules 1 and 2. COM radial distribution functions are also displayed.

At room temperature, dipole moments of the closest molecules tend to be slightly antiparallel, which is probably a result of the repulsive interaction between equal charges. For distances close to the main maximum of the COM radial distribution function $(r=4.6 \AA)$, the most probable orientation for molecules within the first coordination shell is $80^{\circ}$. Similar trends were observed for $\mathrm{MeCN}$, which is also a polar liquid with linear molecules. ${ }^{19}$ Orientational correlations de-

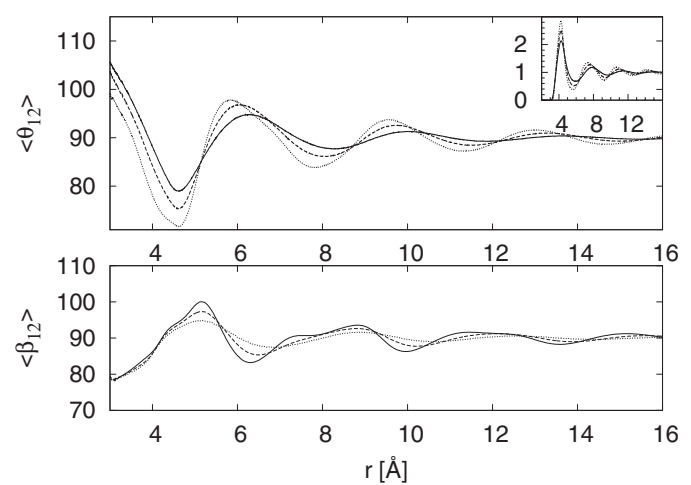

FIG. 1. Average values (in degrees) for $\theta_{12}$ and $\beta_{12}$ (Eqs. (1) and (2), respectively) against distance between centers of mass of molecules 1 and 2 at $298 \mathrm{~K}$ (continuous lines), $178 \mathrm{~K}$ (dashed lines), and $103 \mathrm{~K}$ (dotted lines). Inset: center-of-mass radial distribution functions at the same temperatures. crease rapidly for increasing distances. This behaviour does not qualitatively change upon cooling, as it happens for most pair distribution functions. It is apparent, though, that relative orientations tend to decrease for molecules within the first coordination shell.

We have also evaluated the angle $\beta_{12}$ between the dipole moment of a given molecule 1 and the vector connecting COM of molecules 1 and 2,

$$
\beta_{12}(r)=\cos ^{-1}\left(\mathbf{u}_{\mathbf{1}} \cdot \mathbf{r}\right) .
$$

This function is also shown in Figure 1. At room temperature, a shoulder is apparent for very short distances which is blurred at the lowest temperature under study. This is probably a signature of the increasing bond orientational order in the supercooled state. ${ }^{20}$ At room temperature, and for most molecules within the first coordination shell, $\beta_{12}=100^{\circ}$. This value slightly decreases at lower temperatures, approaching perpendicularity. Then, the projection of the dipole moment onto the direction parallel to the line connecting consecutive molecular COM tends to be fairly small, especially at lower temperatures.

\section{B. Reorientation and rotation}

We analyze dipole reorientation by means of the autocorrelation function

$$
C_{2}(t)=\left\langle P_{2}(\mathbf{u}(\mathbf{t}) \cdot \mathbf{u}(\mathbf{0}))\right\rangle=\left\langle\mathbf{P}_{2}(\cos (\theta(\mathbf{t}))\rangle,\right.
$$

where $\mathbf{u}(\mathbf{t})$ is the unit vector in the direction of a given molecular dipole at a time interval $t$. Then, $\theta(t)$ is the dipole reorientation angle during the same time interval. $P_{2}$ refers to the second Legendre polynomial.

Three dynamic regimes are encountered for $C_{2}(t)$ in the supercooled state, much alike than it was obtained for correlation functions characterizing translational dynamics. Accordingly, the long-time regime or $\alpha$-relaxation can be fitted to a stretched exponential $\left(C_{2}(t)=A \exp \left(-\left(t / \tau_{2}\right)^{\beta}\right)\right)$. Relaxation times $\tau_{2}$ are evaluated by taking into account that $C_{2}\left(\tau_{2}\right)=$ $1 / e$ and they are gathered in Table II. When compared to translational relaxation times $(\tau)$, also listed in Table II, it is apparent that both increase upon cooling and that $\tau>\tau_{2}$ for all temperatures. In addition, the ratio $\tau / \tau_{2}$ remains approximately constant for temperatures higher than $200 \mathrm{~K}$, which proves that they are coupled at high temperatures. For lower temperatures, $\tau$ increases faster than $\tau_{2}$, showing that in this temperature range translational motions become more hindered than reorientational ones. It has been previously shown that the dynamics in the supercooled regime is heterogeneous. ${ }^{21}$ Different properties of translational/rotational dynamical heterogeneities within this temperature range could be at the root of this behaviour. Nevertheless, when fitting $\tau_{2}$ to a power law $\left(\tau_{2} \propto\left(T-T_{c}\right)^{-\gamma}\right)$, the corresponding parameters are $T_{c}$ $=98 \mathrm{~K}$ and $\gamma=1.2$. In consequence, even though the slowing down for the dynamics is much more marked for translation than for reorientation, their associated critical temperatures are very close, in agreement with mode-coupling predictions.

The probability distribution of $\cos \theta$ is displayed in Figure 2 for several time intervals. At $298 \mathrm{~K}$, and for short 
TABLE II. Relaxation times associated with the self-intermediate scattering function $(\tau)$, with $C_{2}(t)\left(\tau_{2}\right)$ and with $C_{\omega}(t)\left(\tau_{J_{k}}\right)$, and translational $(D)$ and rotational $\left(D_{r}\right)$ diffusion coefficients. $I$ is the inertial moment and $k_{B}$ is the Boltzmann constant.

\begin{tabular}{lccccccc}
\hline \hline$T(\mathrm{~K})$ & $\tau(\mathrm{ps})$ & $\tau_{2}(\mathrm{ps})$ & $\tau / \tau_{2}$ & $\tau_{J_{k}}(\mathrm{ps})$ & $D^{\mathrm{a}}\left(\mathrm{cm}^{2} / \mathrm{s}\right)$ & $D_{r}\left(\mathrm{rad}^{2} / \mathrm{ps}\right)$ & $I /\left(k_{B} T \tau_{2} \tau_{J_{k}}\right)$ \\
\hline 298 & 0.53 & 0.18 & 2.9 & 6.3 & $5.69 \times 10^{-5}$ & 0.98 & 5.6 \\
268 & 0.63 & 0.21 & 3.0 & 6.0 & $4.32 \times 10^{-5}$ & 0.83 & 5.7 \\
238 & 0.81 & 0.25 & 3.3 & 5.3 & $2.94 \times 10^{-5}$ & 0.56 & 6.0 \\
218 & 1.03 & 0.29 & 3.6 & 4.9 & $2.17 \times 10^{-5}$ & 0.44 & 6.1 \\
208 & 1.14 & 0.32 & 3.6 & 4.7 & $1.82 \times 10^{-5}$ & 0.40 & 6.1 \\
198 & 1.34 & 0.35 & 3.8 & 4.5 & $1.45 \times 10^{-5}$ & 0.39 & 6.1 \\
178 & 2.01 & 0.46 & 4.3 & 4.0 & $0.94 \times 10^{-5}$ & 0.33 & 5.9 \\
158 & 3.62 & 0.65 & 5.6 & 3.5 & $0.53 \times 10^{-5}$ & 0.18 & 5.4 \\
138 & 8.52 & 1.12 & 7.6 & 2.9 & $0.20 \times 10^{-5}$ & 0.14 & 4.3 \\
123 & 26.7 & 2.21 & 12.1 & 2.5 & $0.80 \times 10^{-6}$ & 0.11 & 2.8 \\
103 & 278. & 12.7 & 21.9 & 2.0 & $0.70 \times 10^{-7}$ & 0.05 & 0.7 \\
\hline \hline
\end{tabular}

${ }^{\mathrm{a}}$ Reference 14.

$t$, the distribution displays a broad maximum corresponding to $\theta \approx 45^{\circ}$. It is also apparent that a randomized distribution is not obtained for $t=1 \mathrm{ps}$, but for $t=2.5 \mathrm{ps,} \mathrm{whereas} \mathrm{at}$ this temperature $\tau_{2}=0.18 \mathrm{ps}$ and $\tau=0.53 \mathrm{ps}$. That is, to obtain a random distribution for the dipole reorientation angle, time intervals much longer than relaxation times, both translational and reorientational, have to be considered. At $103 \mathrm{~K}$, all distributions $\mathrm{P}(\cos \theta)$ display a maximum corresponding to $\theta$ $\approx 25^{\circ}$, which is consistent with a more restricted dynamics upon cooling. This maximum becomes slightly broader and shifted towards larger $\theta$ values (smaller $\cos \theta$ ) as larger time intervals are considered. Nevertheless, even when considering $t>\tau_{2}$, the distribution is still far from being randomized. Larger time intervals satisfying that $t>\tau$ are required in order to obtain a random distribution of reorientational angles.

Our study of the rotational dynamics requires the evaluation of the angular variable

$$
\phi_{k}(t)=\int_{o}^{t} \omega_{k}(s) d s
$$

where $\omega_{k}(s)$ is the angular velocity of a given molecule about the principal axis of inertia $k$ at time $s$. For linear molecules
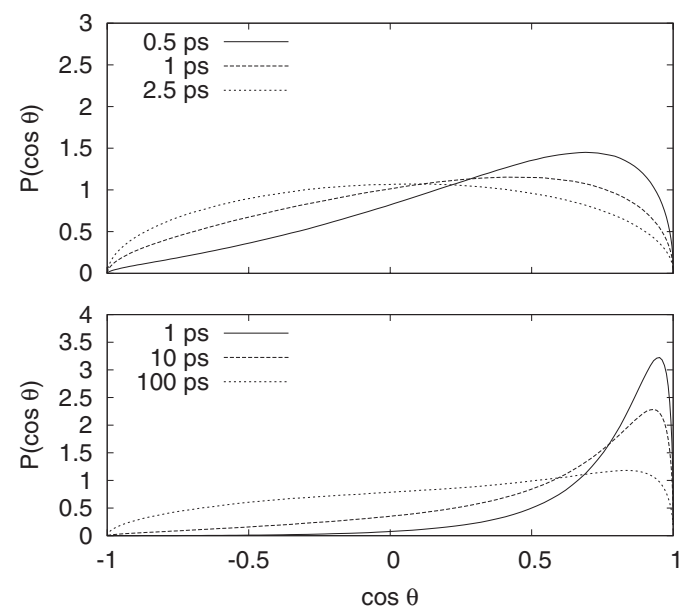

FIG. 2. Probability of $\cos \theta$, being $\theta$ the reorientation angle (Sec. III B) at $298 \mathrm{~K}$ (up) and $103 \mathrm{~K}$ (down) for different time intervals.

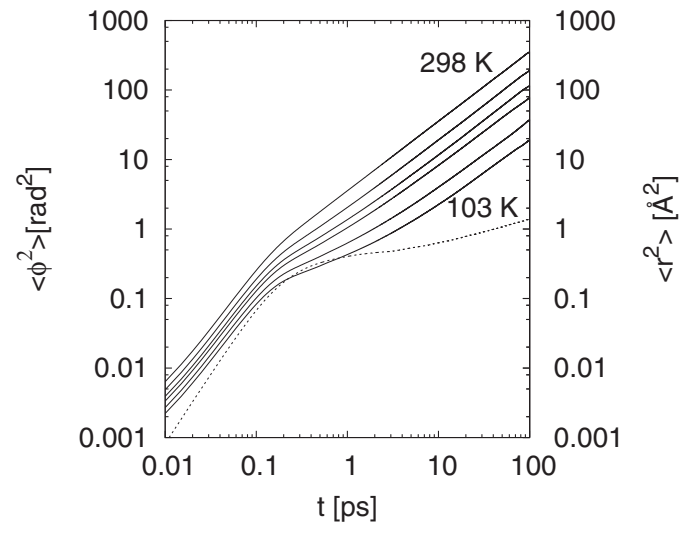

FIG. 3. Rotational mean square displacement $\left\langle\phi^{2}(t)\right\rangle$ (Eq. (5)) at $298 \mathrm{~K}$, $218 \mathrm{~K}, 178 \mathrm{~K}, 158 \mathrm{~K}, 123 \mathrm{~K}$, and $103 \mathrm{~K}$ (up to down, continuous lines). Also shown is the molecular center-of-mass mean square displacement, $\left\langle r^{2}(t)\right\rangle$, at $103 \mathrm{~K}$ (dotted line).

$k=1,2$ because two inertial axes exist, both perpendicular to the molecular dipole moment. The angular displacement $\phi_{k}(t)$ is a two-dimensional vector that plays a similar role in describing the rotational trajectory of a molecule as the displacement vector does for the translational one. Analogous to the mean square displacement, the rotational mean square displacement can be evaluated as

$$
\left\langle\phi^{2}(t)\right\rangle=\frac{1}{2 N}\left\langle\sum_{i} \sum_{k}\left\langle\left|\phi_{i k}(t)-\phi_{i k}(0)\right|^{2}\right\rangle\right\rangle,
$$

where $i$ runs from 1 to $N$, and $k$ runs from 1 to $2 .\left\langle\phi^{2}(t)\right\rangle$ has been displayed in Figure 3 for a set of temperatures ranging between $298 \mathrm{~K}$ and $103 \mathrm{~K}$. The three dynamic regimes observed in previous works ${ }^{9}$ are apparent: $\left\langle\phi^{2}(t)\right\rangle$ $\propto t^{2}$ on a short time scale, $\left\langle\phi^{2}(t)\right\rangle \propto t$ in the long-time diffusive region, and a transition regime between them. The $\mathrm{COM}$ mean square displacement at $103 \mathrm{~K}$ is also shown in Figure 3. It is apparent that the intermediate regime of $\left\langle\phi^{2}(t)\right\rangle$ is less pronounced and extends less on the time scale than the one of $\left\langle r^{2}(t)\right\rangle$. Then, angular trapping is weaker than the one affecting COM dynamics.

From the slope of the diffusive regime, the rotational diffusion coefficient $\left(D_{r}\right)$ can be evaluated as

$$
D_{r}=\lim _{t \rightarrow \infty} \frac{\left\langle\phi^{2}(t)\right\rangle}{4 t} .
$$

As shown in Table II, $D_{r}$ decreases upon cooling by one order of magnitude in the temperature range under study, whereas its translational counterpart $(D)$ decreases by three orders of magnitude. These results were also found for a system of nonpolar molecules. ${ }^{9,10}$ When fitting $D_{r}$ to a power law, a critical temperature of $58 \mathrm{~K}$ is obtained, which is lower than those associated with translational and reorientational relaxation times, and also with translational diffusion coefficients. Then, rotational dynamics about a direction perpendicular to the molecular axis is less hindered than translational dynamics upon cooling, and it is still active when translational dynamics is already frozen.

The Stokes-Einstein-Debye (SED) relation has also been tested in our system. SED states that $D_{r} \tau / T$ takes a constant 


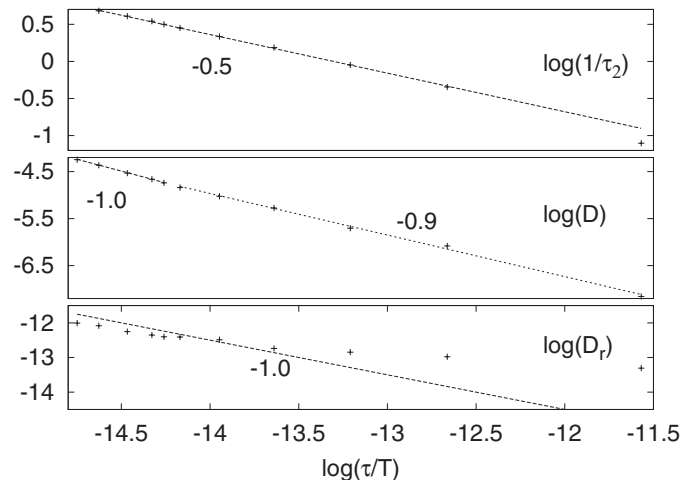

FIG. 4. Breakdown of the Stokes-Einstein and the Stokes-Einstein-Debye relations. Values for the slopes of the straight lines are indicated in the figure.

value, provided that $\tau$ is proportional to the viscosity. The relation was derived for the liquid state. ${ }^{22}$ Figure 4 displays $D_{r}$ against $\tau / T$. SED is fulfilled for $T>158 \mathrm{~K}$ as the results can be well reproduced by a linear fit with slope -1 , but it is apparent that SED does not hold at lower temperatures. When checking the SED relation, $D_{r}$ has frequently been replaced by the inverse of the reorientational relaxation time $\tau_{2}$, which can be experimentally measured. It is shown in Figure 4 that a linear fit with a slope equal to -0.5 reproduces the behaviour for temperatures higher than $123 \mathrm{~K}$. Our results show that in a non-associate dipolar molecular liquid, substitution of $D_{r}$ with $\tau_{2}^{-1}$ is not appropriate because SED does not hold even at high temperatures. This result was obtained for water. ${ }^{6}$ It must be taken into account that these parameters refer to different dynamical processes. $D_{r}$ gives information on the rotation about an axis perpendicular to the molecular dipole, whereas dipole reorientation does not necessarily take place as a result of rotation about a specific inertial axis. In addition, small angular motions of a molecule trapped in the cage made up by its coordination shell molecules contribute to rotational diffusion but are unable to relax dipole orientation.

Results on the $\mathrm{SE}^{23}$ relation are also shown in Figure 4. The relation breaks at higher temperatures than SED does, as it is fulfilled only for temperatures higher than $200 \mathrm{~K}$. A fractional SE relation $\left(D \propto(\tau / T)^{\gamma}\right)$, with an exponent equal to -0.9 , is a better fit at lower temperatures.

We have evaluated the autocorrelation function of angular velocities

$$
C_{\omega_{k}}(t)=\frac{\left\langle\omega_{k}(t) \cdot \omega_{k}(0)\right\rangle}{\left\langle\omega_{k}(0) \cdot \omega_{k}(0)\right\rangle} .
$$

The $C_{\omega}(t)$ function has been obtained by averaging $C_{\omega_{k}}(t)$ over the two degenerate inertial axis. $C_{\omega}(t)$ have been displayed in Figure 5. For each temperature, it is apparent that the decay of $C_{\omega}(t)$ is much faster than the one of $C_{2}(t)$, also displayed in the figure. This was also observed in tethahedral molecules ${ }^{24}$ and it is a necessary condition for a diffusive dynamics. After an initial decay, all $C_{\omega}(t)$ take negative values for $t \ll \tau_{2}$. The change of sign of the angular velocity is a consequence of the collisions of a molecule with other molecules belonging to its coordination shell. When this change takes place, rotation about an inertial axis is still active, but it does not significantly contribute to orientation relaxation. Upon

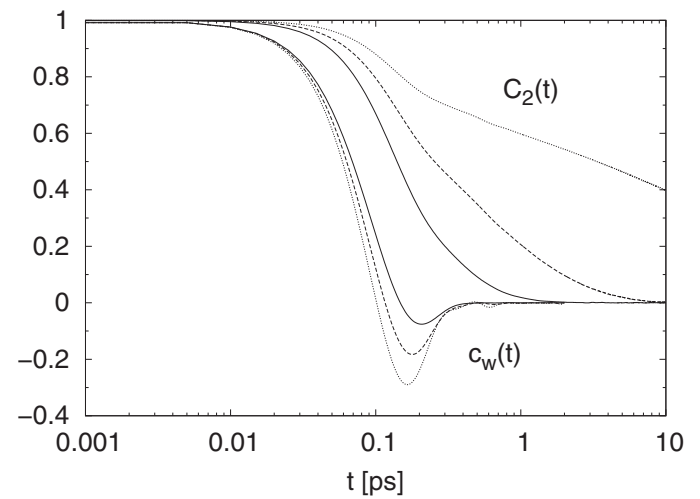

FIG. 5. Angular velocity autocorrelation functions $\left(C_{\omega}(t)\right)$ and reorientational correlation functions $\left(C_{2}(t)\right)$ at $298 \mathrm{~K}$ (continuous lines), $178 \mathrm{~K}$ (dashed lines), and $103 \mathrm{~K}$ (dotted lines).

cooling, collisions with the cage become more important and the $C_{\omega}(t)$ minimum decreases.

By assuming a Langevin equation for the angular momentum, and in the diffusive regime, it has been shown that the reorientational correlation time $\tau_{2}$ and the correlation time for the angular momentum $\tau_{J_{k}}$ are related through ${ }^{25}$

$$
\tau_{2} \tau_{J_{k}}=\frac{I}{6 k_{B} T}
$$

where $I$ is the inertial moment, $k_{B}$ is the Boltzmann constant, and $\tau_{J_{k}}$ can be obtained from

$$
\tau_{J_{k}}=\int_{o}^{\infty} C_{\omega_{k}}(t) d t .
$$

The fulfillment of Eq. (8) can be assessed from results gathered in Table II. At high temperatures, the equation is valid, and only differences smaller than $6 \%$ are observed. For temperatures lower than $158 \mathrm{~K}$, the temperature dependence of $\tau_{2} \tau_{J_{k}}$ is much stronger than that given by Eq. (8), and a generalized diffusion model should be considered. ${ }^{26}$

To get a deeper insight in the coupling between reorientation and translation in the supercooled state, we have seeked for correlations between molecular COM translation and dipole reorientation by evaluating $\left\langle\theta_{01}\right\rangle$. This function returns the average value of the reorientational angle $\theta$ for molecules whose COM has performed a displacement equal to $r_{01}$ during a time interval $t_{01}$. The $\left\langle\theta_{01}\right\rangle$ functions have been displayed in Figure 6 for several time intervals at $103 \mathrm{~K}$. No correlation is observed for short time intervals, but for time intervals larger than $10 \mathrm{ps}$, already in the early $\alpha$-relaxation regime, molecules which have performed COM displacements larger than average have also performed larger than average reorientation angles. This is an evidence of the coupling between reorientation and translation in the longtime regime.

We have also evaluated the function $\left\langle\phi_{01}^{2}\right\rangle$, which returns the angular mean square displacement (Eq. (5)) for molecules with a COM displacement equal to $r_{01}$, during a time interval $t_{01}$. As shown in Figure 7, no correlation is found at $103 \mathrm{~K}$. Then, translation and rotation about an inertial molecular axis are independent processes in the temperature range under study. This uncoupling is reinforced by the aforementioned 


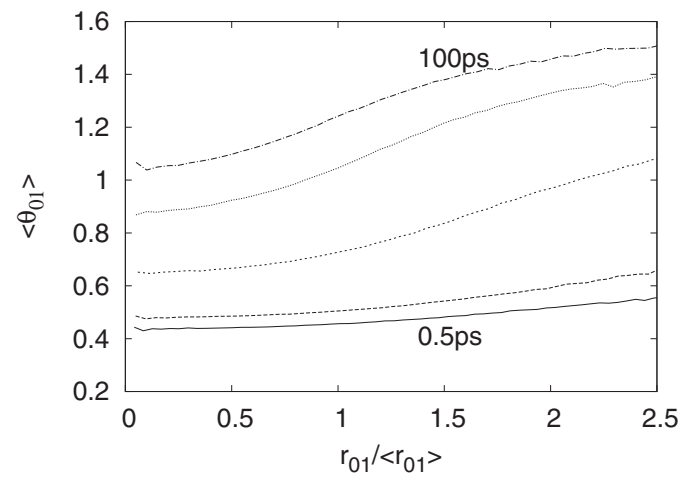

FIG. 6. Correlation between reorientation and translation: $\left\langle\theta_{01}\right\rangle$ (rad) at $103 \mathrm{~K}$ for $t_{01}=100 \mathrm{ps}, 50 \mathrm{ps}, 10 \mathrm{ps}, 1 \mathrm{ps}, 0.5 \mathrm{ps}$ (from up to down).

result that the $D_{r}$ temperature dependence is characterized by a lower critical temperature than that of $\tau$.

It is now widely accepted that dynamical heterogeneities ${ }^{27}$ are crucial in the comprehension of the rich phenomenology of supercooled liquids approaching the glass transition. It was previously demonstrated in a glass-forming Lennard-Jones liquid that the mobile particles formed string-like clusters in the supercooled state, and that these particles had a tendency to move in the string's axis direction. ${ }^{28}$ When analyzing the dynamics of molecular COM in our supercooled system, it was obtained that molecules with different mobilities tend to form clusters. Their geometry depends upon the specific mobility, and the most mobile clusters are characterized by a quasi-linear geometry. ${ }^{21} \mathrm{We}$ have shown in Sec. III A that the molecular dipole tends to be orthogonal to the line connecting consecutive molecular COM, and that this tendency increases upon cooling. This line is coincident with the string's axis at very low temperatures, when heterogeneities arise. Then, according to the results in Ref. 28, the string's axis would be the preferential direction for translation so that a molecule would occupy the former position of its closest molecule along the string, whereas dipole rotation about an inertial axis mainly takes place in a plane perpendicular to that direction. This tendency to orthogonality between the directions of rotational and translational motions would explain the lack of correlation between rotation and translation shown in Figure 7, as well

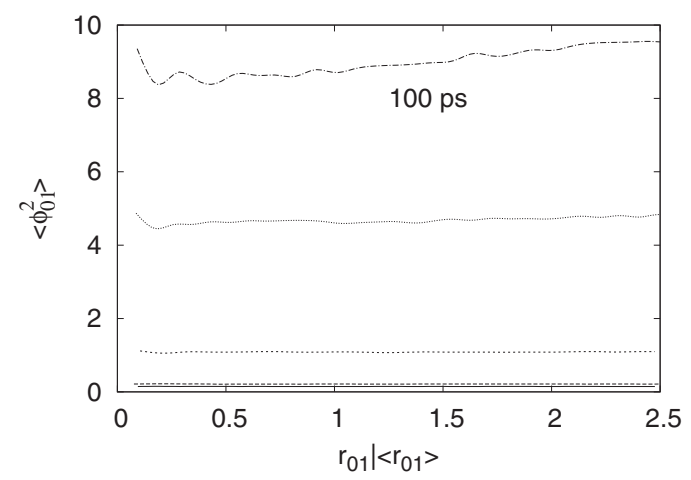

FIG. 7. Correlation between rotation and translation: $\left\langle\phi_{01}^{2}\right\rangle\left(\operatorname{rad}^{2}\right)$ at $103 \mathrm{~K}$ for $t_{01}=100 \mathrm{ps}, 50 \mathrm{ps}, 10 \mathrm{ps}, 1 \mathrm{ps}, 0.5 \mathrm{ps}$ (from up to down). as the result that rotation about an inertial axis is still active when translation is remarkably hindered.

\section{CONCLUDING REMARKS}

Reorientational dynamics has been studied in a supercooled system composed of rigid dipolar molecules. We have obtained that relaxation times for translation are always larger than reorientational ones. Nevertheless, both relaxation times can be fit fairly well to power laws with the same critical temperature, as predicted by the mode-coupling theory. The coupling between dipole reorientation and translation at very low temperatures results in that the most mobile molecules in translation have also performed the largest reorientational angles in the early $\alpha$-relaxation regime. Moreover, time intervals larger than translational relaxation times are required in order to completely randomize the distribution of reorientational angles.

We have investigated rotational dynamics about an axis perpendicular to the molecular dipole, and no significant influence of dipole interactions has been found. Rotational diffusion coefficients have been evaluated from the slope of angular mean square displacements. When comparing with their translational counterparts, we have encountered that translational trapping is stronger than the one affecting rotational dynamics and that translational diffusion is more hindered than the rotational one. In addition, the critical temperature associated with the latter is significantly lower, which means that rotation is still active at temperatures characterized by a hindered translational dynamics. As for the correlations between translation and rotation about an inertial axis, we have found that they are independent dynamic variables in the temperature range under study. The tendency to orthogonality between the directions of rotational and translational motions is at the root of the rationale that we propose for this lack of correlation.

The Stokes-Einstein relation has been checked and we have found that it breaks at a higher temperature than the Stokes-Einstein-Debye relation does. When checking it, we have obtained that substitution of rotational diffusion coefficients by the inverse of reorientational times is not appropriate, as observed for other molecular systems. Small motions originated by angular trapping contribute to rotational diffusion but are unable to relax dipole reorientation.

\section{ACKNOWLEDGMENTS}

Financial support of Dirección General de Investigación Científica y Técnica (DGICYT) (Project FIS2009-13641C02-01) and of Generalitat de Catalunya (Project 2009 SGR1003) is acknowledged.

${ }^{1}$ See, for example, M. T. Cicerone, and M. D. Ediger, J. Chem. Phys. 104, 7210 (1996); D. B. Hall, A. Dhinojwala, and J. M. Torkelson, Phys. Rev. Lett. 79, 103 (1997).

${ }^{2}$ F. Fujara, B. Geil, H. Sillescu, and G. Fleischer, Z. Phys. B: Condens. Matter 88, 195 (1992).

${ }^{3}$ I. Chang and H. Sillescu, J. Phys. Chem. B 101, 8794 (1997).

${ }^{4}$ Z. Zheng, F. Wang, and Y. Han, Phys. Rev. Lett. 107, 065702 (2011). 
${ }^{5}$ T. G. Lombardo, P. G. Debenedetti, and F. H. Stillinger, J. Chem. Phys. 125, 174507 (2006).

${ }^{6}$ S. R. Becker, P. H. Poole, and F. W. Starr, Phys. Rev. Lett. 97, 055901 (2006).

${ }^{7}$ M. G. Mazza, N. Giobambatista, F. W. Starr, and H. E. Stanley, Phys. Rev. Lett. 96, 057803 (2006).

${ }^{8}$ M. G. Mazza, N. Giobambatista, H. E. Stanley, and F. W. Starr, Phys. Rev. E 76, 031203 (2007).

${ }^{9}$ S. Kammerer, W. Kob, and R. Schilling, Phys. Rev. E 56, 5450 (1997).

${ }^{10}$ C. De Michele and D. Leporini, Phys. Rev. E 63, 036702 (2001).

${ }^{11}$ S.-H. Chong and W. Kob, Phys. Rev. Lett. 102, 025702 (2009).

${ }^{12}$ M. P. Allen and D. J. Tildesley, Computer Simulations of Liquids (Clarendon, Oxford, 1987).

${ }^{13}$ R. Palomar and G. Sesé, Phys. Rev. E 75, 011505 (2007).

${ }^{14}$ R. Palomar and G. Sesé, J. Phys. Chem. B 109, 499 (2005).

${ }^{15}$ J. P. Hansen and I. R. McDonald, Theory of Simple Liquids (Academic, London, 1986).

${ }^{16}$ W. Kob in Supercooled Liquids: Advances and Novel Applications, ACS Symposium Series Vol. 676, edited by J. T. Fourkas, D. Kivelson, U. Mohanty, and K. A. Nelson (American Chemical Society, Washington, DC, 1997).
${ }^{17}$ Diffusion coefficients have been evaluated from the long-time slope of COM mean square displacements, by using the Einstein relation $D$ $=\lim _{t \rightarrow \infty}\left\langle r^{2}(t)\right\rangle / 6 t$ (see, for example, Ref. 15).

${ }^{18}$ In the paper, translational relaxation times $(\tau)$ are the times that characterize the long-time regime of the COM incoherent scattering functions such that $F_{s}(k, \tau)=1 / e$.

${ }^{19}$ H. J. Böhm, I. R. McDonald, and P. A. Madden, Mol. Phys. 49, 347 (1983).

${ }^{20}$ H. Tanaka, J. Stat. Mech.: Theory Exp. 2010, P12001 (2010).

${ }^{21}$ R. Palomar and G. Sesé, J. Chem. Phys. 129, 064505 (2008).

${ }^{22}$ P. E. Egelstaff, An Introduction to the Liquid State (Clarendon, Oxford, 1992).

${ }^{23}$ A. Einstein, Investigations on the Theory of the Brownian Motion (Dover, New York, 1956).

${ }^{24}$ R. Rey, J. Phys. Chem. B 112, 344 (2008).

${ }^{25}$ P. S. Hubbard, Phys. Rev. 131, 1155 (1963).

${ }^{26}$ R. G. Gordon, J. Chem. Phys. 44, 1830 (1966).

${ }^{27}$ L. Berthier, G. Biroli, J. P. Bouchaud, L. Cipelletti, and W. van Saarloos, Dynamical Heterogeneities in Glasses, Colloids, and Granular Media, International Series of Monographs on Physics, Vol. 150 (Oxford University Press, 2011)

${ }^{28}$ C. Donati, S. C. Glotzer, P. H. Poole, W. Kob, and S. J. Plimpton, Phys. Rev. E 60, 3107 (1999). 